\title{
Endolymphatic Hydrops Reversal following Acetazolamide Therapy: Demonstration with Delayed Intravenous Contrast- Enhanced 3D-FLAIR MRI
}

\author{
(D) A.R. Sepahdari, N. Vorasubin, G. Ishiyama, and A. Ishiyama
}

\begin{abstract}
SUMMARY: Endolymphatic hydrops, the primary pathologic alteration in Menière disease, can be visualized by using delayed intravenous contrast-enhanced 3D-FLAIR MR imaging. It is not known whether MR imaging-demonstrable changes of hydrops fluctuate with disease activity or are fixed. We describe the results of baseline and posttreatment MR imaging studies in a group of subjects with Menière disease with hydrops who were treated with acetazolamide. Seven subjects with untreated Menière disease with MR imaging evidence of hydrops had repeat MR imaging during acetazolamide treatment. Symptoms and imaging findings were assessed at each time point. Five subjects showed symptom improvement, of whom 3 had improvement or resolution of hydrops. One subject had recurrent symptoms with recurrent hydrops after discontinuing therapy. Two had unchanged hydrops despite symptom improvement. Subjects with unchanged symptoms had unchanged hydrops. Hydrops reversal may be seen with acetazolamide treatment in Menière disease. MR imaging may provide an additional biomarker of disease.
\end{abstract}

ABBREVIATIONS: hT2WI-FLAIR = heavily T2-weighted 3D FLAIR; MD = Menière disease; SPACE = sampling perfection with application-optimized contrasts by using different flip angle evolutions

M eniere disease $(\mathrm{MD})$ is an incompletely understood condition characterized by symptoms of hearing loss, aural fullness, vertigo, and tinnitus. ${ }^{1}$ The clinical phenotype and severity of symptoms vary among patients, and symptoms commonly fluctuate, typically presenting with fluctuating hearing loss that progresses and vertigo spells that eventually are associated with peripheral vestibular damage. ${ }^{2}$ A variety of treatments are available, including low-salt diet, oral diuretics, intratympanic steroid injection, and endolymphatic sac shunt surgery. ${ }^{3}$ The efficacy of these treatments has not been proved, and the mechanism of their effects is speculative.

MD is characterized by endolymphatic hydrops (ie, ballooning of the endolymphatic system), which has been described in human temporal bone postmortem studies. ${ }^{4,5}$ Recent advances in

Received March 9, 2015; accepted after revision May 25.

From the Departments of Radiological Sciences (A.R.S.), Head and Neck Surgery (N.V., A.I.), and Neurology (G.I.), David Geffen School of Medicine, University of California, Los Angeles, Los Angeles, California.

Drs Vorasubin and Sepahdari contributed equally to this work.

Preliminary results of this work were previously presented at: Annual Meeting of the American Otological Society, May 16-17, 2014; Las Vegas, Nevada.

Please address correspondence to Ali Sepahdari, MD, Department of Radiological Sciences, David Geffen School of Medicine, University of California, Los

Angeles, 757 Westwood Plaza, Suite 1621D, Los Angeles, CA 90095; e-mail: alisepahdari@gmail.com

三 Indicates article with supplemental on-line table.

http://dx.doi.org/10.3174/ajnr.A4462
MR imaging technology have allowed in vivo imaging of endolymphatic hydrops, which has the potential to advance our understanding of the natural history of the disease and assess changes in response to treatment. Several groups have confirmed the ability of delayed intravenous contrast-enhanced MR imaging to detect hydrops, ${ }^{6-8}$ but no prior studies have assessed serial changes in hydrops during diuretic treatment by using delayed intravenous contrast-enhanced MR imaging, to our knowledge. One study that followed patients treated with betahistine by using intratympanic contrast-enhanced MR imaging did not show hydrops reversal despite symptomatic improvement. ${ }^{9}$ Another study by using contrast administered through the eustachian tube showed decreased endolymphatic space size following endolymphatic sac decompression in a small group of patients. ${ }^{10}$

Our goal was to evaluate baseline and posttreatment MR imaging changes of endolymphatic hydrops in a group of patients with MD treated with oral acetazolamide, with the goal of developing a noninvasive objective biomarker for disease activity.

\section{MATERIALS AND METHODS \\ Subjects}

This institutional review board-approved study was performed with a waiver of informed consent and a waiver of Health Insurance Portability and Accountability Act authorization. A clinical data base of 356 subjects with hearing loss and/or vestibular 


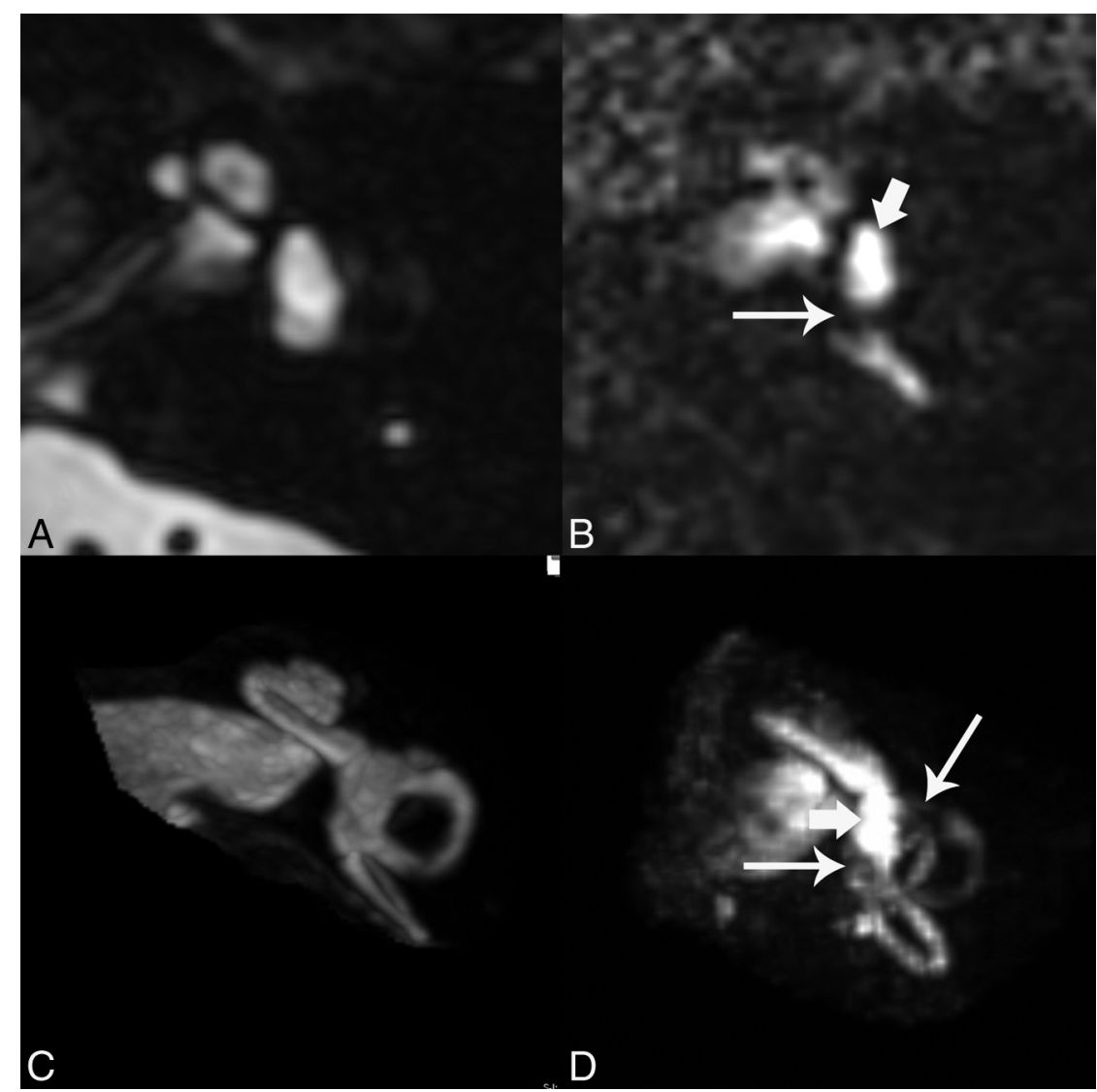

FIG 1. Normal appearance of cisternographic T2 (T2 SPACE) and heavily T2-weighted 3D FLAIR. A, Axial T2 SPACE source image through the midmodiolar level shows normal bright signal of fluid in the vestibule. Both endolymph and perilymph are bright by this technique. B, Axial hT2WI-FLAIR source image at the same level shows predominantly bright perilymphatic fluid in this part of the vestibule (short arrow), with a small signal void reflecting normal endolymphatic space near the ampulla of the posterior semicircular canal (long arrow). C, 3D maximum intensity projection of the T2 SPACE sequence shows the cochlea, vestibule, and semicircular canals in the same image, all with normal bright fluid signal. D, 3D MIP of the hT2WI-FLAIR sequence shows 2 small signal voids in the vestibule reflective of normal endolymphatic spaces (long arrows). The vestibule is predominantly filled with bright perilymph (short arrow).

symptoms imaged with hydrops-protocol MR imaging (4-hour delayed double-dose intravenous contrast-enhanced MR imaging using 3D-FLAIR sequences) was queried. Seven subjects met the following inclusion criteria: 1) clinical diagnosis of definite or probable MD, as determined by American Academy of Otolaryngology-Head and Neck Surgery guidelines ${ }^{11}$;2) pretreatment MR imaging showing evidence of endolymphatic hydrops; and 3) posttreatment MR imaging during treatment with acetazolamide. Acetazolamide treatment was initiated at a dose of $250 \mathrm{mg}$ by mouth daily. Treatment was continued indefinitely if there was a response.

\section{Imaging}

MR imaging was performed on a 3T Magnetom Skyra unit (Siemens, Erlangen, Germany) by using a 16-channel head and neck coil, 4 hours following an intravenous injection of 0.2 $\mathrm{mmol} / \mathrm{kg}$ of either gadopentetate dimeglumine (Magnevist; Bayer HealthCare Pharmaceuticals, Wayne, New Jersey) or gadobutrol (Gadavist; Bayer Schering Pharma, Berlin, Germany). Scanning consisted of a "cisternographic" heavily T2-weighted $3 \mathrm{D}$ turbo spin-echo sequence (sampling perfection with application-optimized contrasts by using different flip angle evolutions
[T2 SPACE]; Siemens) and a heavily T2weighted 3D FLAIR sequence (hT2WIFLAIR). ${ }^{12}$ All sequences were performed as volumetric axial scans through the inner ear and internal auditory canals. The hT2WI-FLAIR sequence was performed with the following parameters: section thickness, $0.8 \mathrm{~mm}$; TR/TE, 9000/534 ms; TI, 2350 ms; number of averages, 2; echo-train length, 144; flip angle, 120; matrix, $320 \times 260$; FOV, $200 \times 167 \mathrm{~mm}$; acquisition time, 6 minutes 45 seconds.

The hT2WI-FLAIR images normally show bright signal in the perilymph due to accumulation of dilute contrast in this space and dark signal in the endolymph, which remains protected from the contrast due to the presence of tight junctions. The T2 SPACE sequence shows bright signal within both endolymph and perilymph. The hT2WIFLAIR images were reformatted in an axial plane parallel to the lateral semicircular canal to standardize interpretation and were also reconstructed as 3D maximum intensity projections. Figure 1 shows the normal appearance of T2 SPACE and hT2WI-FLAIR images. The images were evaluated with respect to the presence of endolymphatic hydrops. The qualitative criteria described by Baráth et $\mathrm{al}^{7}$ were used to assess the $2 \mathrm{D}$ images for the presence of hydrops in the vestibule. 3D MIPs were also reviewed, and the criteria described by Sepahdari et $\mathrm{al}^{8}$ were used to assess these images for hydrops in the vestibule. Cochlear hydrops was not specifically assessed because it was not thought that the cochlea is consistently evaluated due to the limitations of spatial resolution.

\section{RESULTS}

Of the 7 patients who met the inclusion criteria, all were diagnosed with definite MD. One of these patients was clinically diagnosed as having delayed endolymphatic hydrops, a subset of MD. All patients had unilateral clinical symptoms and unilateral hydrops on MR imaging. Five experienced improvement in symptoms; 3 of these 5 also showed either complete or partial reversal of hydrops (Fig 2). Two patients with symptomatic improvement did not have any change in hydrops. The 2 patients whose symptoms did not reverse showed unchanged hydrops. Clinical data are summarized in the On-line Table. Patient 3 required a dose increase to $250 \mathrm{mg}$ twice daily before achieving a response. Patient 4 was only able to tolerate a dose of $62.5 \mathrm{mg}$ daily due to medical comorbidities and did not have a response. Patient 1 had a return of vertigo and hydrops after attempting discontinuation of acet- 
azolamide, and his symptoms improved after resuming treatment. A subsequent attempt to taper to $125 \mathrm{mg}$ daily resulted in a return of vertigo.

The average duration of symptoms before initial MR imaging in all patients was 7.4 months. The average duration of treatment before follow-up imaging was 4.5 months. The average follow-up period was 19 months. Among the 3 patients whose symptoms and hydrops reversed, 2 continued acetazol- amide treatment and were followed for an average of 9.5 months without recurrent symptoms. One patient discontinued acetazolamide and experienced recurrent symptoms with recurrent MR imaging evidence of hydrops (Fig 3 ). The 2 patients who had symptom improvement without hydrops reversal were imaged an average of 4 months after initiating treatment. The 3 patients with hydrops reversal were imaged an average of 6 months after treatment.

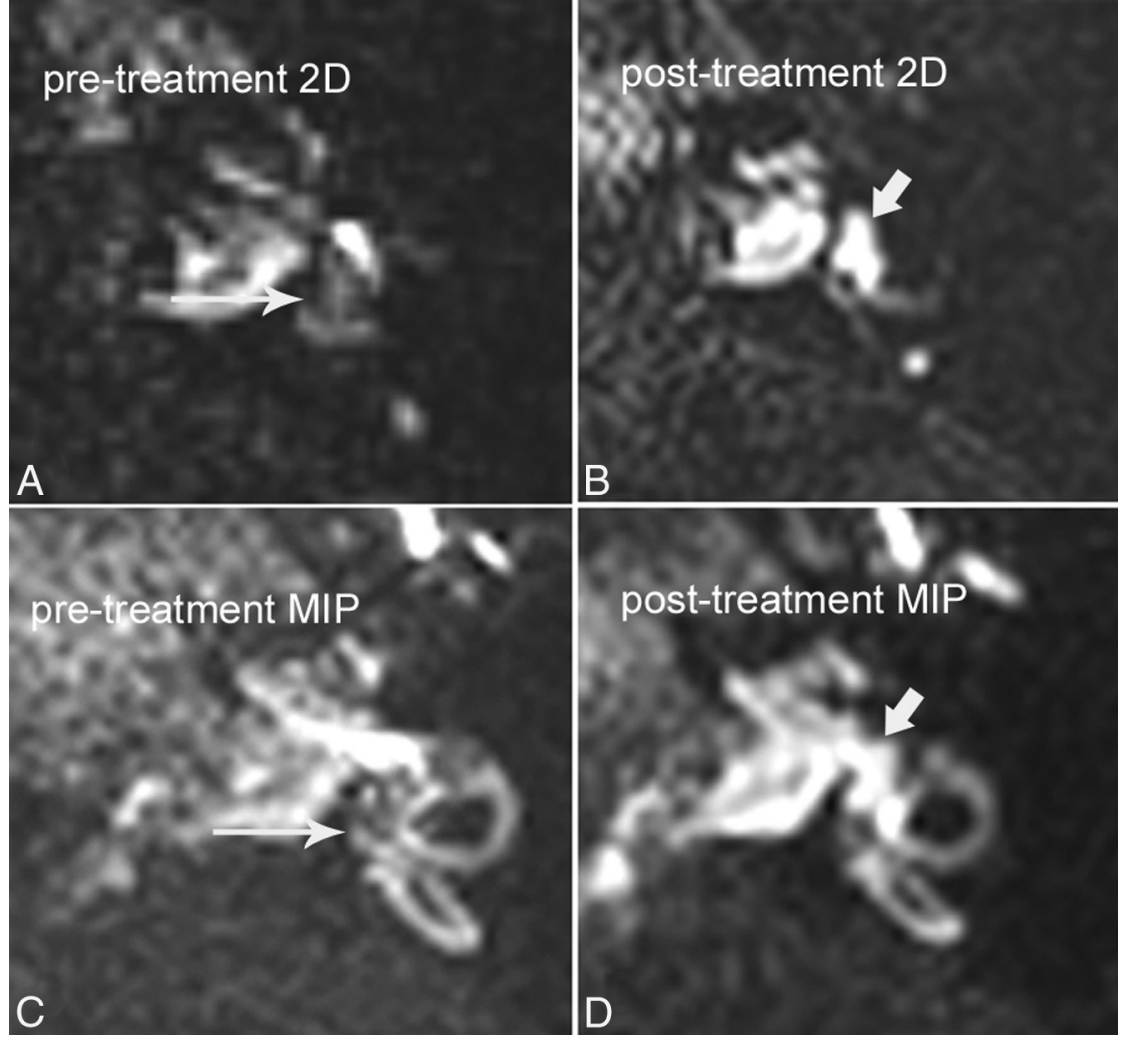

FIG 2. A 42-year-old man with grade I vestibular hydrops, with reversal of symptoms and reversal of hydrops after treatment with acetazolamide. A, Pretreatment axial hT2WIFLAIR source image through the vestibule shows dilated endolymphatic spaces effacing the vestibular perilymph (long arrow). Compare with the normal appearance of the inner ear in Fig 1B. B, Posttreatment axial hT2WI-FLAIR source image through the vestibule shows interval resolution of endolymphatic hydrops, with normal bright perilymph signal in the vestibule (short arrow). C, Pretreatment hT2WI-FLAIR 3D MIP shows dilated endolymphatic spaces effacing $>50 \%$ of the vestibule (long arrow). D, Posttreatment hT2WI-FLAIR 3D MIP shows interval resolution of hydrops, with normal bright perilymph (short arrow) occupying $>50 \%$ of the vestibule.

\section{DISCUSSION}

Delayed intravenous contrast-enhanced MR imaging is an emerging technique for evaluating patients with MD. The ability to visualize hydrops with this technique has been confirmed by multiple groups on different continents. ${ }^{6-8,13}$ To date, however, no strong clinical application has been established for this technique, and existing research is mostly limited to showing a connection between a clinical diagnosis of MD and imaging evidence of hydrops.

By showing reversibility of hydrops, we demonstrate that MR imaging can provide a biomarker of disease. This is critical for evaluating the effects of various treatments, particularly when exploring new therapies. Symptoms are known to fluctuate in MD and hence do not provide a sufficient marker of treatment effectiveness on their own. Audiometric testing and vestibular evoked myogenic potentials offer added objective measures of disease severity in MD but may also be confounded by other variables, such as presbyacusis or additional pathology such as otosclerosis. ${ }^{14}$ MR imaging visualization of hydrops provides a unique, quantifiable marker of a direct effect of the disease.

One patient experienced reversal of

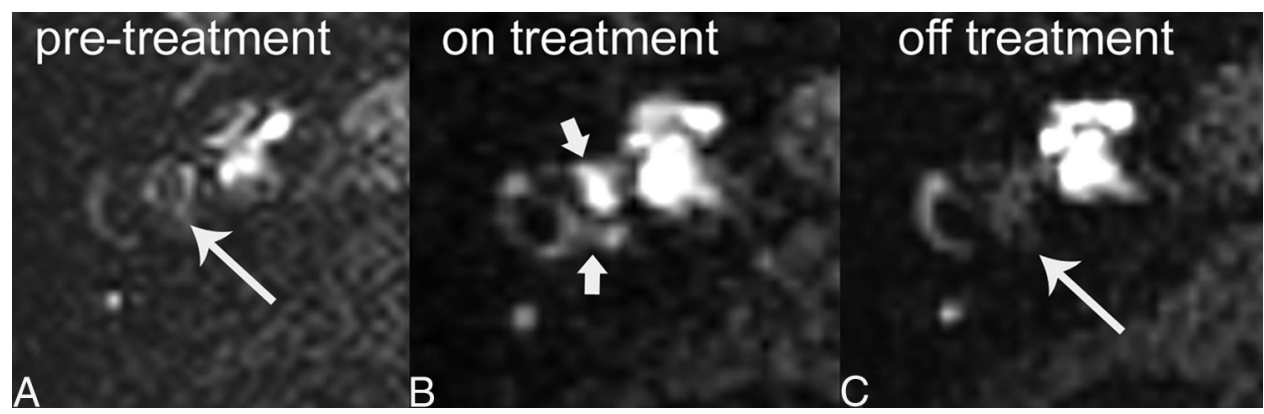

FIG 3. A 72-year-old man with hydrops responsive to acetazolamide and recurrence after discontinuation of treatment. $A$, Pretreatment axial hT2WI-FLAIR source image through the vestibule shows marked hydrops with complete effacement of the normal bright vestibular perilymphatic fluid by dilated, dark endolymphatic space (long arrow). B, Posttreatment axial hT2WI-FLAIR, during treatment with acetazolamide, shows reversal of hydrops. Normal bright perilymphatic fluid is now visible (short arrows). C, Following discontinuation of acetazolamide and recurrence of symptoms, follow-up axial hT2WI-FLAIR image shows recurrence of hydrops (long arrow). 
symptoms and reversal of MR imaging-evident hydrops while on acetazolamide and re-emergence of symptoms with re-emergence of hydrops after stopping diuretics. Although this was just a single case, it shows a promising connection among acetazolamide treatment, symptoms, and hydrops.

Notably, 2 of 5 patients in our study who had symptom reversal did not have hydrops reversal. The reasons for this result are unclear because the small number of subjects does not permit a robust statistical analysis. However, factors such as the duration of disease before treatment and the length of time between treatment and repeat imaging may influence the results. Alternatively, the symptomatic improvement in these patients may have been a coincidence related to normal symptom fluctuation rather than an effect of the treatment. Rather than considering this phenomenon as a limitation of MR imaging, we view it as evidence that MR imaging provides unique information that is not duplicated by eliciting a clinical history from the patient.

The primary limitation of this study is the small number of subjects. Although a high volume of hydrops-protocol MRI is performed at our institution, only a small number of subjects met the inclusion criteria for this analysis. Despite the small number of subjects, the results provide support for the use of pre- and posttreatment hydrops-protocol MR imaging in prospective investigations of treatments for MD. There are also inherent technical limitations of the MR imaging technique and limits of image interpretation. Although our MR imaging technique has equal or superior resolution to other techniques reported in the literature, further technical improvements are needed to confidently visualize the entire membranous labyrinth. The method of image assessment, categorizing each ear as normal, grade I hydrops, or grade II hydrops, may be insensitive to subtle changes among scans. Further advancements in image acquisition and image interpretation techniques are needed to fully realize the potential of hydrops imaging.

\section{CONCLUSIONS}

Pilot data from a group of patients imaged with hydrops-protocol MR imaging before and during acetazolamide treatment show that endolymphatic hydrops is a reversible feature of Menière disease. Hydrops-protocol MR imaging provides a unique biomarker of disease in MD and may be valuable in assessing the effects of treatment.

\section{REFERENCES}

1. Andrews JC, Honrubia V. Menière's disease. In: Baloh RW, Halmagyi GM, eds. Disorders of the Vestibular System. New York: Oxford University Press; 1996: 300-17

2. Paparella MM, Sajjadi $H$. The natural history of Menière's disease. In: Harris JP, ed. Menière's Disease. Amsterdam: Kugler Publications; 1999:29-40

3. Chung JW, Fayad J, Linthicum F, et al. Histopathology after endolymphatic sac surgery for Ménière's syndrome. Otol Neurotol 2011;32:660-64 CrossRef Medline

4. Paparella MM. Pathogenesis and pathophysiology of Menière's disease. Acta Otolaryngol Suppl 1991;485:26-35 Medline

5. Merchant SN, Adams JC, Nadol JB Jr. Pathophysiology of Menière's syndrome: are symptoms caused by endolymphatic hydrops? Otol Neurotol 2005;26:74-81 CrossRef Medline

6. Pyykko I, Nakashima T, Yoshida T, et al. Menière's disease: a reappraisal supported by a variable latency of symptoms and the MRI visualisation of endolymphatic hydrops. BMJ Open 2013;3:pii: e001555 CrossRef Medline

7. Baráth K, Schuknecht B, Naldi AM, et al. Detection and grading of endolymphatic hydrops in Menière disease using MR imaging. AJNR Am J Neuroradiol 2014;35:1387-92 CrossRef Medline

8. Sepahdari AR, Ishiyama G, Vorasubin N, et al. Delayed intravenous contrast-enhanced 3D FLAIR MRI in Menière's disease: correlation of quantitative measures of endolymphatic hydrops with hearing. Clin Imaging 2015;39:26-31 CrossRef Medline

9. Gürkov R, Flatz W, Keeser D, et al. Effect of standard-dose betahistine on endolymphatic hydrops: an MRI pilot study. Eur Arch Otorhinolaryngol 2013;270:1231-35 CrossRef Medline

10. Liu F, Huang W, Meng X, et al. Comparison of noninvasive evaluation of endolymphatic hydrops in Menière's disease and endolymphatic space in healthy volunteers using magnetic resonance imaging. Acta Otolaryngol 2012;132:234-40 CrossRef Medline

11. Committee on Hearing and Equilibrium guidelines for the diagnosis and evaluation of therapy in Menière's disease: American Academy of Otolaryngology-Head and Neck Foundation, Inc. Otolaryngol Head Neck Surg 1995;113:181-85 CrossRef Medline

12. Naganawa S, Yamazaki M, Kawai H, et al. Visualization of endolymphatic hydrops in Ménière's disease with single-dose intravenous gadolinium-based contrast media using heavily T(2)-weighted 3D-FLAIR. Magn Reson Med Sci 2010;9:237-42 CrossRef Medline

13. Naganawa $S$, Nakashima T. Visualization of endolymphatic hydrops with MR imaging in patients with Ménière's disease and related pathologies: current status of its methods and clinical significance. Jpn J Radiol 2014;32:191-204 CrossRef Medline

14. Mukaida T, Sone M, Yoshida T, et al. Magnetic resonance imaging evaluation of endolymphatic hydrops in cases with otosclerosis. Otol Neurotol 2015;36:1146-50 CrossRef Medline 